\title{
Italique Italique
}

Poésie italienne de la Renaissance

XX $\mid 2017$

Varia

\section{Genere bucolico poesia pastorale. Le metamorfosi dell'egloga nel Quattrocento}

\section{Antonia Tissoni Benvenuti}

\section{(2) OpenEdition}

1 Journals

\section{Edizione digitale}

URL: http://journals.openedition.org/italique/448

DOI: $10.4000 /$ italique.448

ISSN: 1663-4438

\section{Editore}

Librairie Droz

\section{Edizione cartacea}

Data di pubblicazione: 1 ottobre 2017

Paginazione: 13-31

ISBN: 978-2-600-05818-6

ISSN: 1423-3983

\section{Notizia bibliografica digitale}

Antonia Tissoni Benvenuti, « Genere bucolico poesia pastorale. Le metamorfosi dell'egloga nel

Quattrocento », Italique [Online], XX | 2017, online dal 01 octobre 2019, consultato il 21 janvier 2020

URL : http://journals.openedition.org/italique/448; DOI : 10.4000/italique.448 
Antonia Tissoni Benvenuti

G E N E R E B U CO L I C O

POESIA PASTORALE.

LE METAMORFOS DELL'EGLOGA

NEL Q U A T R O C E N T O 



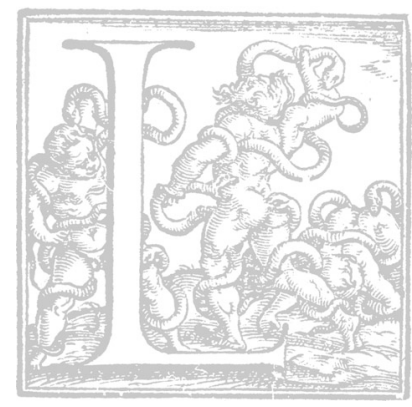

'opera di Virgilio per secoli è stata paradigma di tre generi stilisticamente ben definiti: la bucolica, stile umile; le Georgiche, stile medio; l'Eneide, stile alto. I tre stili erano schematizzati nella medievale Rota Virgilii. Non si può non pensare anche alla miniatura di Simone Martini nel Virgilio di Petrarca. L'insegnamento del latino aveva inizio con la bucolica di

Virgilio: di qui la sua universale conoscenza e la sua migrazione nei generi piu disparati. E. R. Curtius lo ba detto molto bene:

Dal primo secolo dell'impero romano fino all'epoca di Goethe, lo studio del latino è sempre incominciato con la lettura della prima ecloga; non si esagera affermando che manca una chiave della tradizione letteraria europea a chi non abbia presente questo piccolo poemetto. ${ }^{\mathrm{I}}$

Lo studio delle ecloghe era accompagnato dal commento di Servio, che specificava nelle pagine iniziali $i$ caratteri del genere. Quando l'imitazione, o meglio la ri-creazione dell'antico diventa inevitabile, l'analisi di Servio resta fondamentale, tanto che la sua caratterizzazione può essere ripresa quasi alla lettera dal Calmeta - e siamo alla fine del Quattrocento se non all'inizio del secolo successivo - nel suo scritto Dell'antichità del buccolico verso e che circonstanze all'egloga si convengono. ${ }^{2}$ Il punto di vista del Calmeta in questo saggio è a mio parere utile per capire problemi e soluzioni della bucolica quattrocentesca. Avremo modo di citarlo più volte. Rileggiamo l'esordio, p. I2:

Molti antichi e moderni auttori, massimamente quelli che la buccolica del Mantovan poeta espongono, circa l'origine del buccolico verso molte opinioni adducono, le quali, per non essere molto al proposito nostro necessarie, per fuggir prolissità pretermitteremo, solo restando contenti alla conclusione di Servio commentatore, il quale, poi che varie opinioni ha addutte, afferma il buccolico verso esser antichissimo e aver origine da quella prima età nella quale generalmente la pastoral vita era essercitata, $\mathrm{e}$ però la elocuzione in essa deve esser umile e semplice, per ripresentar quella prima età aurea, nella quale gli uomini, quasi tutti pastori, della dolce semplicità si dilettavano. Onde, avendo gli scrittori tre figure o vero caratteri di dire, umile, medio e grandiloquo, l'egloga solo dell'umile si 
debbe contentare, né in essa alcuna urbanità, arguzia, o vero declamativa sonorità si debbe ritrovare; anzi, tutte le azioni, tutte le comparazioni e faccende devono essere di cose rustiche.

Ricordiamo brevemente altri caratteri dell'ecloga latina classica. Poteva essere monodica o dialogica, in entrambi $i$ casi con variazioni, o meglio sottocategorie; $i$ contenuti dovevano essere, come s'è visto, adatti a pastori e alle loro vicende, perlopiù amorose. L'autore inseriva di norma $i$ nomi reali dei luoghi, ma quelli degli interlocutori dovevano essere fittizi, e tradizionalmente pastorali. Da Virgilio in poi, potevano anche essere espressi sotto il velamen o cortex pastorale contenuti politici, politico-profetici, spesso per noi di difficile se non impossibile decodificazione. ${ }^{3}$ Da un punto di vista retorico, le figure proprie dell'ecloga devono essere semplici, umili: figure di ripetizione (dall'allitterazione all'anafora ad altri procedimenti seriali); $i$ paragoni, gli adunata, la gratulatio (lode del canto), pure con un procedimento seriale, e spesso introdotti da qualis...talis. I tre ultimi casi citati devono veicolare immagini naturali adeguate al mondo bucolico, come sottolineano Servio e il Calmeta.

Per secoli la bucolica è stata l'unico genere in cui per statuto venivano inserite evocazioni o brevi descrizioni di scene naturali. E quindi topoi connotativi dell'egloga sono il locus amoenus, il luogo pastorale per eccellenza, dove solitamente si svolge l'azione; l'invocazione alla natura, come unico possibile interlocutore di un canto d'amore; l'età dell'oro, un'età perduta ma che può ritornare (il topos è usato anche per encomi politici), alla quale il mondo pastorale appartiene, come scrive Calmeta nel brano citato sopra. Tratti costitutivi di un altrove innocente, sereno: l'egloga viene cosi a veicolare l'eterna utopistica tensione dell'nomo verso un paradiso perduto o futuro. Ed è per noi difficile capire perché proprio questo idillio bucolico, quasi per antitesi, in una sorta di ossimoro, possa anche diventare il linguaggio in cui si celano fatti politici o biografici a volte drammaticamente coinvolgenti per l'autore. Una caratteristica dell'egloga di cui Calmeta non fa parola, e che per noi è molto spesso indecifrabile. ${ }^{4}$ La frequente incomprensione o il travisamento di queste allusioni, perdute per i lettori lontani nel tempo, non ha impedito che il fascino del cortex sia stato fecondo per secoli nei molti testi pastorali della letteratura europea. 
Nel passaggio al volgare, $i$ caratteri connotativi dell'ecloga classica vengono conservati. Dal punto di vista metrico prevale la terza rima, con possibili inserzioni di altri metri. Insieme alla terzina, la rima sdrucciola diventerà una caratteristica dell'egloga. Il Calmeta in proposito, notando con Servio la presenza di dattili nella bucolica classica, ritiene che l'uso della rima sdrucciola in volgare nasca appunto dal desiderio di imitare quel ritmo e distinguere cosi la terzina bucolica da quella di stile alto, pp. I3-I4:

conoscendo che i Latini e ancor più i Greci ne' versi bucolici locano certi dattili a loro proprie sedi, per tenere in bassezza il bucolico verso che dall'eroico sia differente, così fu trovato da' volgari di mettere il dattilo nel fin del verso, che volgarmente si chiama sdrucciolo, accioché da' grandiloqui ternari fosse lo stil de l'egloga differente.

Calmeta cita poi l'uso pervasivo ma non inelegante che ne fa Sannazaro, p. I4:

Il che dal Sannazaro nella sua egloga tanto si frequenta, che è grandissimo miracolo come sotto tale imperio possi con tal continuazione sì dolcemente i concetti della sua mente esprimere.

Com'è noto, si attribuisce a Boccaccio la scelta della terzina, da lui usata per le due egloghe inserite nell'Ameto: le prime egloghe volgari. Il prosimetro di Boccaccio, come poi vedremo, è estremamente importante nella storia della letteratura pastorale, ma la scelta della terzina sembra casuale, dato che tutti $i$ testi in versi dell'Ameto sono in terza rima. La terzina è un metro dalle molte possibilità stilistiche; viene usata anche per l'elegia, la satira, la poesia didascalica, le traduzioni dei comici latini e molto altro. Che questo sia il metro prevalente dell'egloga è per noi ovvio. Poteva essere scelto un altro metro?

C'è un caso curioso di possibile incarnazione metrica dell'egloga: il madrigale. Nel'Trattato de li ritmi volgari Gidino da Sommacampagna riprende da Antonio da Tempo ${ }^{6}$ la forma latina mandrialis $e$ il conseguente rapporto con $i$ pastori: lo marigale in lingua latina vocatur mandrialis, quasi cosa uscita da la mandra de le pegore. E aggiunge, p. I33: 
imperzò che questo modo de rithimare primamente venne da li pastori inamorati, li quali, sì come homeni rustici e grossi, per compiacere a le loro femine rusticane cominciarono a compillare parole grosse, e quelle cantavano ne le pive loro con grosso modo ma naturalmente; quamvis deo che li moderni facieno li marighali loro con più sotile e più ligiadre parole.

Fin qui Gidino traduce Antonio da Tempo. Ma, mentre gli esempi di madrigale citati da Antonio da Tempo non presentano nessun colore bucolico, per Gidino è stretta la connessione del madrigale con l'ecloga virgiliana: $i$ suoi sette esempi delle possibili forme metriche non sono che traduzioni di frammenti delle ecloghe di Virgilio, adattate al metro in questione. Traduzioni letterali, non con parole grosse: niente di rusticale come ci si potrebbe attendere. Si veda il primo esempio, relativo a un madrighale tutto undenario, un mosaico dalla prima di Virgilio:

Titiro lento manifestamente

De l'alto fago ti riposi all'ombra,

Dove may vento e pioggia non t'ingombra.

Quivi sì dolze canta la tua piva,

Che per tuo canto per tutte le spiaggie

Fanno leticia le piante selvaggie.

E noi tapini se vogliamo bere,

A gli affri fiumi correr ne convene,

Con grande angoscia e con dogliose pene.

Or dunque, Melibeo, le pere incalma,

Fa riconciar le vite e li arboselli

A ciò che 'l non ci franga i sterpi felli.?

E cosi negli altri esempi citati, o meglio, creati da Gidino. Non mi risulta che esistano madrigali, e tanto meno collane di madrigali, cosi strettamente bucolici; ma non ne sono troppo lontani i madrigali 52 e 54 di Petrarca, con la pastorella nel primo e il faggio nel secondo. Sono del resto quattro madrigali anche i versi IOI-32 della II egloga di Sannazaro. $E$ che da questa fisionomia bucolica del madrigale, oltre che dai noti testi di Alberti e Giusto, venga l'idea di Boiardo del Mandrialis cantu dimetro rithmo intercalari, ${ }^{8}$ presente nella microsezione pastorale del suo canzoniere?9 Anche l'unico altro madrigale suo, I 8, condivide col genere bucolico il topos dell'invocazione alla natura, impersonata nei vaghi augelletti. 
La polimetria, a partire dall'Arzocchi, ${ }^{\text {IO }}$ diventa uno dei caratteri connotativi dell'egloga; si vedano tra $i$ primi casi, La notte torna e l'aria e 'l ciel s'annera, di Giusto, in cui alle terzine e alla frottola si uniscono sequenze simili a stanze di canzone; ${ }^{\text {I }}$ o la ballata inserita nel Corymbus dell'Alberti. L'Alberti occupa un posto di grande importanza all'inizio della storia dell'egloga volgare, anche per la sua codificazione metrica. Ricordiamo che l'elegia Mirtia, come Udite monti alpestri di Giusto e poi il Mandrialis di Boiardo importano dall'ottava ecloga di Virgilio l'uso di versi intercalari, che in volgare diventano una sorta di ritornello. Una caratteristica dell'ecloga classica, non dell'elegia: quando, all'inizio di questi generi volgari, il confine tra egloga ed elegia era piuttosto labile.

Non a caso il Calmeta si sofferma con giudizi positivi solo sull'Alberti

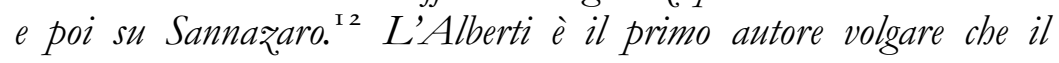
Calmeta cita:

Da questa auttorità adunque i poeti volgari pigliando essempio, quegli che hanno avuto giudicio si sono sforzati nelle loro egloghe il pastoral decoro servare, e con agreste comparazione a' lor poemi porgere ornamento. Il primo che in questo stile abbia alcuna perfezione dimostrato fu Battista Alberti fiorentino, il quale in versi toscani le sue egloghe esprimendo, ben dimostrò della poetica arte non essere ignaro. ${ }^{13}$

Il gindizio del Calmeta fa supporre che le egloghe scritte dall'Alberti fossero molte di più delle due che abbiamo. Ma nelle pochissime rime sue pervenuteci, il côté pastorale è molto presente in sé, non come cortex di altri significati: dall'egloga dialogata Tyrsis, fino a una sestina, Io miro, Amor, la terra e fiumi e l'onde ( $x$, che Gorni giustamente definisce "sestina pastorale"); e si aggiunga, come s'è visto, anche l'inserzione della ballata nel Corymbus (che io ritengo un'egloga, non un'elegia pastorale).

Esempi tutti che Sannazaro aveva certo presenti: si pensi alle sestine dell'Arcadia: la sestina doppia dialogata IV e la sestina VII. Sannazaro, com'è noto, dopo aver passato in rassegna gli autori del genere bucolico nella XI prosa, afferma di essere stato superato soltanto da Tirsi - che con grande probabilità è l'Alberti stesso - grazie agli strumenti da lui usati: ciò̀ la sua lingua toscana. ${ }^{\mathrm{I}}$

Mentre la polimetria, da Arzocchi a Sannazaro trionfa, Boiardo, amante quanti altri mai degli artifici metrici nei Carmina e negli 
Amorum Libri, nelle sue Pastorale è estremamente fedele alla terza rima, al punto che l'unica frottola inserita nella $V$ è piuttosto una terz̧a rima con rimalmezzo, rispetta cioè le rime incatenate; e anche la rima sdrucciola è rara. L'unico artificio, autorizzato del resto da Virgilio, è il verso intercalare nei due canti della IV pastorale.

Per una trasposizione in volgare della bucolica, ebbe fortuna anche l'ottava: a Firenze, e nel filone rusticale soprattutto. La scelta rusticale è rifiutata dal Calmeta, che la attribuiva a una pericolosa accentuazione dello stile umile, p. Iз:

essendo la natura del parlar volgare di sorte che, quando è dalla sentenza rimoto, facilmente in bassezza e vernaculo sapore incorre.

Ma qualche sfumatura in direqione rusticale nei paragoni (di ascendenza ovidiana) era già nell'egloga VIII dell'Ameto: «tu se' lucente e chiara più che 'l vetro / e assa' dolce più ch'uva matura» ( $v v$. 6I-62) ${ }^{\text {Is }}$ e più ancora nell'egloga Tyrsis di Alberti: «O saporita dal viso rosato / che hai quelli occhiazzi più bei che 'l mio toro»» $(v v .19-2 I) \cdot{ }^{16}$

Il filone rusticale o nenciale, di probabile origine teocritea, rimase un gioco ristretto all'ambiente fiorentino; il giudizio negativo del Calmeta in proposito è anche sottinteso nelle sue lodi a Sannazaro, al quale «del bucolico verso indubitatamente si può dar la palma», appunto perché è riuscito a tenersi in un giusto medio.

Questo per quanto attiene alla forma metrica dell'egloga volgare. In molte egloghe, anche in quelle volgari, come s'è detto, il mondo pastorale è stato per secoli solo velamen o cortex, cioè un travestimento pastorale di contenuti altri e volutamente nascosti. Ma in altri casi, a mio parere storicamente piu fecondi - le egloghe dell'Alberti ne sono un sicuro esempio -, il mondo pastorale interessa in sé: ed è in sostanza dal cortex che avranno origine $i$ molti testi pastorali della letteratura europea. ${ }^{17}$

Nel genere lirico bisognerà attendere il pieno Cinquecento per trovare testi di esplicita poesia pastorale nei canzonieri; ma figure tipicamente bucoliche si incontrano molto presto. Non si può prescindere da Petrarca, onnipresente poi in seguito. La lettura di Petrarca delle ecloghe virgiliane, e di conseguenza la sua scrittura bucolica latina, è totalmente allegorica anche nel caso di scene naturali. ${ }^{8}$ Ma Petrarca è tutt'altro che insensibile al fascino del cortex pastorale, che riaffiora 
nelle sue rime, a volte in traduzione. Si veda, per esempio, "onde discende / dagli altissimi monti maggior l'ombra», che ricalca il celebre «maioresque cadunt altis de montibus umbrae» (Ecl. I 83). Oltre a questi versi, la canzone $\mathrm{Ne}$ la stagion che 'l ciel rapido inchina (Rvf 50), accoglie altri ricordi bucolici, come il pastore nella terza stanza, con gli ineludibili faggi. ${ }^{\text {'9 }}$

$E$ c'è molto altro. Come non ricordare "Chiare fresche e dolci acque / $[. .$.$] gentil ramo [...] / herba et fior [...] / aere sacro, sereno [...] / date$ udienza insieme alle dolenti mie parole estreme» ( $\operatorname{Rvf}$ I26): una sorta di canzone pastorale, quasi completamente in settenari, boschereccia e rozza come è definita nel congedo. In questo caso Petrarca riprende il topos dell'invocazione alla natura: in Virgilio sono innumerevoli $i$ casi in cui il canto del pastore ha come teatro la natura, testimone immota, o partecipe (come nel mito di Orfeo), oppure invocata perché ascolti. $^{20}$

Quest'ultimo topos è fortunatissimo nelle rime del Quattrocento, e non solo; è quasi superfluo citare Odite monti alpestri li mei versi di Giusto; ${ }^{21}$ il sonetto Ombrosa selva, che il mio dolo ascolti (II 47) 0 Ecco la pastorela mena al piano (III 32) ${ }^{22}$ di Boiardo (e versi inseriti in altre sue rime, come voi, monti alpestri, oditi il mio martire, II 40, 4; Odite selve e prendavi pietade nella canzone II 44,6I). Aggiungiamo anche Limpido fonte che sovente ascolti, accolto da Mauro tra le Rime disperse di Sannazaro. ${ }^{23}$ E si potrebbe continuare.

Troviamo il medesimo topos, con evidente ricordo petrarchesco, anche nel lamento amoroso di Praseldo, dal I libro dell'Inamoramento de Orlando, (I XII I9):

«Odeti fior, e voi selve,» dicia

«Poi che quella crudel più non me ascolta:

Diati odienza alla sventura mia!

Tu, sol, che hai mo' de il ciel la note tolta,

Voi, chiare stelle, e luna che vai via,

Oditi il mio dolor solo una volta:

Che in queste voce extreme hagio a fenire

Con cruda morte il longo mio martire!

C'è poi anche il polizianesco Udite selve mie dolci parole, dall'Orfeo: ma è inserito in un'egloga, pur in un momento di metamorfosi, come vedremo. 
Più avanti nel tempo, come s'è detto, la poesia pastorale diventerà una componente costante del genere lirico: si pensi alle rime del Bandello (testi come il sonetto $L X$, o la sestina pastorale CXXIX), ${ }^{24}$ a Bernardo Tasso, e a tanti altri.

Abbiamo ricordato l'Orfeo di Poliziano, la prima importante migrazione dell'egloga verso la scena teatrale. Ė un dato di fatto che la prima parte dell'Orfeo sia un'egloga dialogata polimetrica: terzine da 17 a 53, ballata (la canzona di Aristeo) 54-87; ottave (anche dialogate) 88-I27 e canto finale di Aristeo in forma di madrigale. Segue poi la narrazione del mito. Ed è altrettanto indubbio che l'opera sia stata scritta per essere rappresentata: probabilmente per un banchetto cardinalizio.

Ma perché ambientare il mito in un contesto pastorale? Si dirà: Aristeo nelle Georgiche è detto pastor (IV 3I7) e tanto potrebbe bastare. A mio parere la spiegazione è un po' più complessa. Notiamo che i testi teatrali pastoral-mitologici del Quattrocento non imitano né la tragedia né la commedia classiche: questo in un'epoca in cui la creazione letteraria doveva obbligatoriamente rifarsi a modelli antichi, far rinascere $i$ generi antichi. Solo un umanista del livello di Poliziano poteva occuparsi dell'esistenza e delle caratteristiche del poco noto terzo genere teatrale, il satiresco o satirico; ne aveva scritto piu volte, individuandone l'esempio nel Ciclope di Euripide, e, a mio parere, aveva cercato di ricreare il genere satirico con la sua Fabula di Orfeo. ${ }^{25}$

Per gli autori seguenti, non altrettanto dotti, è stato certo importante l'esempio polizianesco, e per tutti sono stati fondamentali $i$ trattati di architettura, dove si descrivono $i$ teatri e le scene per $i$ tre generi. E se Vitruvio si limita a citare le tre scene, ${ }^{26}$ l'Alberti - e secondo me il fatto è determinante - aggiunge la connotazione dei relativi tre generi letterari. Per il terzo genere fa un esplicito riferimento ai pastori:

E poiché la poesia drammatica si divideva in tre generi: tragico, che rappresentava le sventure de' tiranni; comico, che narrava le preoccupazioni e gli affanni dei padri di famiglia; satiresco, che cantava le bellezze della campagna e gli amori dei pastori: non mancavano teatri provvisti di una macchina girevole, tale da presentare senza perdita di tempo una facciata dipinta, mostrando un atrio, una casa, oppure un bosco, secondo la convenienza della rappresentazione. ${ }^{27}$

La precisazione dell'Alberti è ripresa a fine secolo, con ulteriori particolari, dal ferrarese Pellegrino Prisciani: 
Et essendo mo che nel theatro se exerciteno tre facte de poeti - tragici, li quali recitano le miserie de tyranni; comici, che representano li pensieri, affanni et travaglie de patri de famiglia; satirici, li quali cantano et representano la dolceza et piacere de le campagne et ville, li amori et inamoramenti de pastori - per ciò li ornati de cadauna de queste scene fra sé serano disimili [...] per satyrici bisogna adornarla de arbori, spelunche, silve, monti et altre simile parte agreste. ${ }^{28}$

Queste teorizzazioni, insieme all'esempio della Fabula di Orpheo con il suo esordio strettamente bucolico, sono state a mio parere determinanti per lo sviluppo del terzo genere - pastorale, boschereccio, satirico o come vogliamo chiamarlo - cioè di opere che non vogliono essere né tragedia né commedia: il genere più amato del teatro di corte. ${ }^{29} \mathrm{E}$ dato che comparivano sulla scena $i$ pastori, poteva anche essere facile definire questi testi egloga, oppure Egloga o vero pasturale, come il testo del Bellincioni, scritto a Milano per il conte di Caiazzo (in ottave alla toscana, con una frottola all'inizio e una barzelletta in fine). ${ }^{30}$ Calmeta nella trattazione sull'egloga non parla di questo teatro pastorale, ma nella Vita di Serafino Aquilano, a proposito della corte milanese al tempo di Beatrice, scrive:

mai non passava mese che da loro o egloga o comedia o tragedia o altro novo spettaculo o representazione non si aspettasse. ${ }^{3 \mathrm{I}}$

Dove è chiaro che con egloga, in serie con comedia $e$ tragedia, sono indicati i testi del terzo genere: come il Cefalo del Correggio, la Pasitea di Gasparo Visconti e tanti altri spettacoli di corte. Teatro di corte, appunto: anche il rapporto con questo tipo di pubblico è stato secondo me determinante per la prevalenza nel Quattrocento di nuovi testi di questo terzo genere teatrale: storie mitologiche in contesti pastorali, perché negli dei, posti in quell'altrove idillico della tradizione bucolica, nella loro superiorità e libertà morale, i signori piacevolmente si riconoscevano.

Ė interessante vedere con quali espedienti $i$ pastori vengano introdotti in questi testi sostanzialmente mitologici: $i$ pastori ci devono essere, e l'autore deve in qualche modo portarli in scena. E con i pastori vengono introdotte nei testi vere e proprie egloghe dialogate. Nel II atto della Fabula di Cefalo, per esempio, un pastor vecchio trattiene Procri in fuga da Cefalo; poi convoca, con un'ottava in rime sdrucciole, gli altri 
pastori che chiudono l'atto con un'egloga dialogata in funzione di coro. ${ }^{32}$ In seguito i pastori scompaiono, lasciando il campo alle divinità agresti: ninfe, satiri, fauni. Ė infatti un fauno che conclude l'atto III, e anche il suo canto, in terza rima, può essere catalogato come un'egloga monodica. L'inserzione di pastori in altri testi coevi poteva presentare qualche problema di congruenza. Per esempio, nella Pasitea di Gasparo Visconti, in cui il mito di Piramo e Tisbe è agito da personaggi cittadini, e il contesto dei primi atti è più da commedia che da fabula pastorale, due pastori compaiono solo alla fine dell'atto IV con un'egloga dialogata in rime sdrucciole, in cui piangono la morte di Dioneo-Piramo. E non si ripresentano più, neppure nella festa finale in cui Apollo fa risuscitare $i$ due amanti. ${ }^{33}$

Per quanto riguarda un possibile sviluppo narrativo dell'egloga prima dell'Arcadia, notiamo che, sulla falsariga di Boccaccio, un contesto pastorale è comune a tutte le narrazioni mitologiche. L'Ameto dal capitolo VII al XV è in effetti un prosimetro pastorale. Anche se Ameto è un cacciatore più che un pastore, si comporta secondo il canone bucolico:

desteso il corpo sopra il verdeggiante prato, difeso da' raggi solari da piacevoli ombre, così cominciò a cantare. ${ }^{34}$

Il suo canto, Febo salito già a mezzo il cielo (VIII), è un'egloga monodica di invito (topos dell'huc veni), con lodi alla bellezza della donna e promessa di doni, secondo l'esempio ovidiano di Polifemo e Galatea. Attiratipoi dal suono di una sonante sampogna, Ameto e le ninfe «vidono in luogo assai grazioso sedere uno pastore, quivi dalle vicine piagge disceso con la sua mandra, il quale sonava all'ombre recenti》 (X). Il canto del pastore, Teogapen (XI), è una terzarima moraleggiante, cui segue la classica gratulatio bucolica: il canto è stato piacevole

quale a' faticati si presta sopra le verdi erbe il leno sonno, o le chiare fontane e frigide agli assetati. ${ }^{35}$

Segue una rissa tra i pastori; per risolverla viene proposta una gara di canto tra Acaten d'Accademia e Alcesto d'Arcadia, che si realizza nell'egloga dialogica Come Titan del sen dell'Aurora (XIV). L'intervento delle ninfe pone fine alla tenzone. Ma nel prosieguo questo mondo bucolico scompare, in favore di una struttura direi decameroniana: ogni ninfa narra $i$ casi propri in una novella. 
Nel Quattrocento il Boccaccio minore ha molto seguito. Per esempio nel Driadeo di Luca Pulci: ${ }^{36}$ un poemetto in ottave parzialmente pastorale, dalla forte valenza eziologica. E che forse avrebbe voluto essere del tutto bucolico. Questa intenzione bucolica mi pare risulti da alcuni versi della Conclusione (4, 98-99):

Ombre de' boschi mia, frassini e faggi

Che 'l rozzo suono udite de' miei versi,

$[\ldots]$

O sacro monte, il qual le nove Muse

Circondano il bel fonte Pegaseo,

Con lor non farò io cantando scuse;

Non ho servato quel verso amebeo,

E son d'alterni mie opere schiuse.

Nel poemetto, oltre alla presenza di ninfe, satiri e altre divinità, a partire dal terzo libro è protagonista il rozo pastore e d'anni vecchio Tavaiano. Il pastore e Lauro (Lorenzo) cantano in una gara proposta dalla ninfa Estura; ma Lorenzo non usa strumenti pastorali. ${ }^{37}$ Lauro esalta la vita in città, il pastore ripropone tutti i luoghi comuni della bucolica, con un evidente ricordo della prima egloga dell'Arzocchi ${ }^{38}$ e più avanti del canto ovidiano di Polifemo, con $i$ doni offerti e il richiamo alla bellezza dei luoghi pastorali $(3,92)$ :

Noi ce ne andian per campi e per viottole

Co' nostri armenti, in luogo ch'egl'ingrassino:

Secondo i tempi cantiam versi e frottole.

Dormiam la state all'ombra sotto un frassino,

Ne' tufi il verno; sbuchiam gufi e nottole.

Tra le cose positive di Firenze Lauro ricorda Un'accademia, un studio di buccoici $(3,84)$; celebre verso considerato connotativo dell'ambiente culturale mediceo negli anni 60, quando oltre al Driadeo, si aveva il volgarizzamento di Bernardo Pulci della bucolica virgiliana, oltre alla prima versione del Corinto di Lorenzo.

In ogni testo narrativo si incontrano talvolta luoghi ameni, e con loro si assiste a un rapido cambio di genere. Come s'è visto, il cosiddetto locus amoenus, più che un luogo retorico, è il luogo pastorale per antonomasia, la scena in cui si svolge l'azione dell'egloga. La sua evocazione serve anche per attirare l'amato o l'amata, con lo stilema huc ades o huc 
veni. Oppure è effetto della presenza letificante della donna, o scenario tipico nelle descrizioni dell'età dell'oro (come suggeriva il passo di Calmeta citato all'inizio). Un utopico paradiso, evocato spesso, ma anche descritto, nelle narrazioni in versi o in prosa.

La narrativa cavalleresca è piuttosto connotata dalle ambages in una selva indefinita, arturiana. La selva è il teatro dell'avventura, della lotta. Ma di tanto in tanto è interrotta da una radura, da un locus amoenus, e si entra in un altro mondo, talvolta in un mondo magico. Gli esempi sono innumerevoli (Orlando Innamorato I III 32, 37):

Dentro ala selva il Barone amoroso

Guardando intorno se mette a cercare:

Vede un boschetto d'arborscieli ombroso

Che in cierco ha un fiumicel con unde chiare.

$[\ldots]$

Così pensoso gionse a una riviera

De una acqua viva, cristallina e pura:

Tutti li fior che mostra primavera

Avea quivi dipinto la Natura

E faceano ombra sopra a quella riva

Un fagio, un pino et una verda oliva.

Cosi Boiardo all'inizio del suo poema, ma i casi analoghi si susseguono. Non mi dilungo con altri esempi; basti ricordare il capovolgimento operato da Ariosto, quando appunto nel locus amoenus pastorale Orlando incontra il dramma che lo condurrà alla pazzia. Altro caso celebre di inserzione bucolica nella narrativa cavalleresca è l'episodio tassesco di Erminia tra i pastori: il luogo pastorale è sempre presentato come oasi di pace.

Ma per la trasmigrazione di questo scenario bucolico dell'egloga al genere narrativo basti citare l'Arcadia, ${ }^{39}$ che si apre appunto con l'insistita descrizione di un locus amoenus. Un locus amoenus manieristico, eccessivo, dove non c'è solo l'albero ombroso o un boschetto adorno, ma ben 13 alberi diversi e spesso, come in Boccaccio, con la loro implicazione mitologica. E altri luoghi simili si incontrano più avanti nel prosimetro.

Con Sannazaro siamo nell'età d'oro della letteratura pastorale. Il primo lustro degli anni'so vede infatti una diffusione della moda bucolica in tutt'Italia; l'egloga esce dalle scuole, non è limitata a singoli 
interventi, diventa endemica: a Ferrara, le Pastorale di Boiardo (composte nei primi anni'8o, durante la guerra con Venezia alla quale partecipò Alfonso duca di Calabria e a lui dedicate); $;^{40}$ a Firenze la cosiddetta stampa Miscomini del febbraio 1482 (che fa conoscere a un più vasto pubblico anche egloghe vecchie di qualche decennio); ${ }^{4 \mathrm{I}}$ a Napoli Sannazaro e compagni. ${ }^{42}$ E aggiungerei $i$ due incunaboli che rilanciano la fortuna del prosimetro di Boccaccio, l'Ameto: la princeps del I478, a Roma, e la successiva stampa del'79 a Treviso. E noto che alcune egloghe di Sannazaro erano nate, e pare proprio che fossero diffuse, prima della loro inserzione nel prosimetro. Non so quanto abbiano influito su Sannazaro i fatti citati, insieme al suo viaggio a Ferrara con Alfonso nei primi anni '80. Forse questa alluvione di egloghe e, in aggiunta, la fortuna editoriale dell'Ameto, ha propiziato l'idea di inserire rime pastorali in una narrazione in prosa, anch'essa pastorale?

Non mi soffermo sull'Arcadia: ascolteremo in proposito studiosi ben più esperti. L'Arcadia rappresenta il culmine della letteratura pastorale italiana del Quattrocento. Le egloghe tradizionali sembrano tracimare in una prosa bucolica: talvolta sono vere e proprie egloghe in prosa. L'Arcadia, insieme al romanzo ellenistico (penso soprattutto a Dafni e Cloe), promuoverà la fortuna della narrazione pastorale per molti secoli in tutte le letterature europee.

Antonia Tissoni Benvenuti 
I. E.R. Curtius, Letteratura europea e Medio Evo latino, a cura di R. Antonelli, Firenze, Nuova Italia i 9922, p. 2 I 4 . Questa è la prima traduzione italiana. L'opera uscì a Berna nel I 948 e fu pochi anni dopo stroncata da Croce; forse per questo in Italia, a differenza che nel resto del mondo, non venne subito tradotta.

2. Vincenzo Calmeta, Prose e lettere edite e inedite (con due appendici di altri inediti), a cura di C. Grayson, Bologna, Commissione per i testi di lingua, I959, pp. I 2-I4.

3. Si veda Servii Grammatici qui feruntur in Vergilii bucolica et georgica commentaria, recensuit G. Thilo. Lipsiae i 887, III I, pp. I-4. Servio, dopo aver scritto «qualitas autem haec est, scilicet humilis charactern, aver discusso della presenza del dattilo (che Calmeta riprenderà più avanti) e ammesso che Teocrito, a differenza di Virgilio ubique simplex est, aggiunge "personae [...] rusticae sunt et simplicitate gaudentes: unde nihil in his urbanum, nihil declamatorium invenitur; sed ex re rustica sunt omnia negotia, comparationes et si qua sunt alia».

4. Petrarca, com'è noto, scriveva al fratello, inviandogli un’egloga: «Sed quoniam id genus est quod, nisi ex ipso qui condidit auditum, intelligi non possit, ne te inutiliter fatiges, primo quid dicam, deinde quid intendam brevibus explicabo» (Fam. x 4, I 2). Un personale sforzo di decodificazione dell'egloga quattrocentesca nel recente M. Santagata, Pastorale modenese. Boiardo, i poeti e la lotta politica, Bologna, Il Mulino, 2016.

5. Gidino da Sommacampagna, Trattato dei ritmi volgari in un codice del Sec. XIV della Biblioteca Capitolare di Verona, or posto in luce per M.r Gio. Batt. C. Giuliari, Bologna, Romagnoli, I870 (edizione fotomeccanica per la Commissione per i testi di lingua, I968).

6. Antonio Da Tempo, Delle rime volgari, trattato [...] per cura di G. Grion, Bologna i 869 (anastatica, Bologna, Forni, i 970).

7. Gidino da Sommacampagna, Trattato, pp. 1 35-36. I versi di Virgilio interessati sono: «Tityre tu patulae recubans sub tegmine fagi / silvestrem tenui musam meditaris avena» (I-2), «tu, Tityre, lentus in umbra / formosam resonare doces Amaryllida silvas» (3-4), «Ipsae te, Tityre, pinus / ipsi te fontes, ipsa haec arbusta vocabant» (38-39), «At nos hinc alii sitientis ibimus Afros» (63), «Insere nunc, Meliboee, piros, pone ordine vitis» (73).

8. Zanato traduce «Madrigale in forma di canzone a doppio metro e parole rima intercalari».

9. La sezione riguarda i testi 39-48 del secondo libro del canzoniere. Si vedano i relativi commenti nell'edizione a cura di T. Zanato, promossa dal "Centro studi Matteo Maria Boiardo" di Scandiano, uscita nel 20 I 2 a Novara per Interlinea.

ı०. Soprattutto nella prima egloga, la più imitata. È ormai certa la cronologia arzocchiana; si veda Francesco Arzocchi, Egloghe, Edizione critica e commento a cura di S. Fornasiero, Bologna, Commissione per i testi di lingua, I995. 
I I. Stanze di canzone o forme possibili di madrigale? Se ne veda l'edizione e l'analisi metrica di I. Pantani, Il polimetro pastorale di Giusto de' Conti, in La poesia pastorale del Rinascimento, a cura di S. Carrai, Padova, Antenore, I998, pp. I-5 5. In attesa dell'edizione critica delle rime di Giusto, dello stesso Pantani, si può vedere la princeps, stampata a Bologna nel 1472 e accessibile online, piuttosto che l'edizione Vitetti (Lanciano, Carabba, I933).

I 2. Altri sono solo nominati: «Dopo di lui [Alberti] sono stati molti altri $<$ Francesco Arsocchi> sanese, Lorenzo Medice, Girolamo Benivieni, Bernardo Pulci, Jacopo Fiorino, Tebaldeo, il Corso, Serafino e molti altri» (p. I 3). Il nome dell'Arzocchi è stato integrato da Grayson, forse sulla base della probabile conoscenza della stampa Miscomini da parte del Calmeta.

i 3. Calmeta, Prose e lettere, cit., p. i 3.

14. Mi pare un dato sicuro; la supremazia è legata allo strumento - uno arco fortissimo - usato da Tirsi: cioè la lingua (si veda G. Velli, Tra lettura e creazione: Sannazaro, Alfieri, Foscolo, Padova, Antenore, 1983, pp. 5-8).

i s. Giovanni Boccaccio, Comedia delle ninfe fiorentine (Ameto), edizione critica per cura di A.E. Quaglio, Firenze, Sansoni, 1963, p. 29.

I6. Il testo è definito da Gorni come il «primo consapevole esperimento di poesia rusticale del Quattrocento». (Leon Battista Alberti, Rime e versioni poetiche, edizione critica e commento a cura di G. Gorni, Milano-Napoli, Ricciardi, i975, p. 63).

17. Toglierei dal novero il genere romanzo "pastorella", anche se E. Faral, La pastourelle, in «Romania», 49, I923, pp. 204-59, in polemica con chi la considerava poesia popolare, ha visto proprio nello stile umile unito alla presenza di pastori, o appunto di una pastorella, l'elemento decisivo per ascrivere al genere bucolico testi che sono chiaramente comico-satirici rispetto a quel contesto.

I 8. Si veda V. Fera, La petrarchesca 'selva d'Arpino' (Buc. Carm. X 2I3-32I), in «Esperienze Letterarie», Ix, I984, pp. 55-66.

I9. A questo proposito notiamo - dato che le statistiche sono di moda e così anche la povera letteratura pensa di essere à la page - che il faggio, alla cui ombra, com'è noto, siede Titiro nel primo verso della prima ecloga di Virgilio («Titire tu patulae recubans sub tegmine fagi»), compare ben 8 volte nei $R v f$, contro le tre occorrenze ciascuno di abeti, pini e olive; prescindiamo dalla palma e dal lauro, che comportano ben altri significati.

20. Il topos era già migrato nell'elegia classica; cfr. Properzio I i 8: «Vos eritis testes, si quos habet arbor amores, / fagus et Arcadio pinus amica deo».

2 I. Nella Mirtia dell'Alberti, Udite e nostri lacrimosi canti, l'appello non è rivolto alla natura.

22. Con la traduzione da Virgilio «vegendo il sol calare e l'ora tarda / e fumar l'alte vile di luntano» (3-4). 
23. Iacobo Sannazaro, Opere volgari, a cura di A. Mauro, Bari, Laterza, ig6r, p. 229.

24. Matteo Bandello, Rime, Edizione e commenti a cura di M. Danzi, Modena, Panini, I989.

25. Ho raccolto tutta la documentazione relativa in $L$ 'Orfeo del Poliziano con il testo critico dell'originale e delle successive forme teatrali, Padova, Antenore, $1986\left(2^{\circ}\right.$ edizione 2000).

26. Vitruvio descrive in questo modo la scena del terzo genere: «satyricae [scaenae] vero ornantur arboribus, speluncis, montibus, reliquisque agrestibus rebus in topicelis speciem deformati». Così nella princeps (IGI I0346), senza note tipografiche.

27. «Cumque in theatro triplex poetarum genus versaretur, tragicum, qui tyrannorum miserias recitarent; comicum, qui patrum familias curas et sollecitudines explicarent; satyricum, qui ruris amoenitates pastorumque amores cantarent, non deerat ubi versatili machina evestigio frons porrigeretur expictus et apparet seu atrium seu casa seu etiam silva, prout is condiceret fabulisque ageretur», cfr. Leon Battista Alberti, De re aedificatoria. L'architettura, Testo latino e traduzione a cura di G. Orlandi. Introduzione e note di P. Portoghesi, Milano, Il Polifilo, I 966, pp. 737-38. Cito la traduzione dell'Orlandi.

28. Pellegrino Prisciani, Spectacula, a cura di D. Aguzzi Barbagli, Modena, Panini, 1992, p. 46.

29. Dell'imbarazzo degli autori teatrali di fronte a questo genere, fanno fede il rifacimento settentrionale dell'Orfeo polizianesco (sia o no di Boiardo), col titolo di Orphei Tragoedia, e quanto si legge nel prologo della Fabula de Cefalo: «Non vi do questa già per comedìa, / ché in tutto non se observa il modo loro; / né voglio la credati tragedìa, / se ben de ninfe ge vedreti il coro: / Fabula o istroria, quale ella se sia, / io vi la dono, e non per precio d'oro», Teatro del Quattrocento. Le corti padane, a cura di A. Tissoni Benvenuti e M.P. Mussini Sacchi, Torino, UTET, I983, p. 2 Iо.

30. Teatro del Quattrocento, cit., pp. 263-75.

3 I. Vita del facondo poeta vulgare Serafino Aquilano, in Calmeta, Prose e lettere, cit., p. 7 I.

32. Teatro del Quattrocento, cit., p. 229.

33. Questo tipo di teatro di corte era connesso sempre a circostanze festose: di qui la scelta inevitabile del lieto fine, anche contro una tradizione classica opposta. E così abbiamo il coro finale delle baccanti ebbre nell'Orfeo (Poliziano è troppo 'classico' per far rinascere Euridice); abbiamo invece nel Cefalo di Nicolò da Correggio la resurrezione di Procris per opera della dea Diana; nella Pasitea di Gasparo Visconti pure la resurrezione dei due amanti per opera di Apollo. In questi e altri casi con grande uso del deus ex machina: uso che poi diverrà generale, quasi obbligato nel seguente teatro di corte europeo 
34. Boccaccio, Comedia delle ninfe fiorentine (Ameto), cit., p. 27.

35. Boccaccio, Comedia delle ninfe fiorentine (Ameto), cit., p. 46.

36. Lo dobbiamo leggere ancora nella infelice edizione di P.E. Giudici, Lanciano, Carabba i 9i6. Sui Pulci si vedano i molti lavori di Stefano Carrai, e in particolare il capitolo Alle origini della bucolica rinascimentale: Lorenzo e l'umanesimo dei fratelli Pulci, nel suo volume I precetti di Parnaso. Metrica e generi poetici nel Rinascimento italiano, Roma, Bulzoni, I999.

37. Infatti Estura dice: «Tu con la cetra, e tu sampogna e canta; / I' me n'andrò con quel che me' si vanta» $(3,78)$.

38. La rima frottole:nottole è in Arzocchi i 26:28:30. Ricordiamo che uno dei testimoni manoscritti delle egloghe di Arzocchi (Parma, Pal. 2508) è di mano di Luigi Pulci.

39. Si veda l'edizione di Vecce, Roma, Carocci, 2013.

40. Si veda la recente edizione a cura di C. Montagnani, Novara, Interlinea, 20 I 9 , promossa dal Centro studi M.M. Boiardo di Scandiano.

4I. Ne abbiamo anche una ristampa anastatica, Roma, Vecchiarelli, 2009. Oltre all'Arzocchi, la stampa raccoglie la traduzione in terzine di Bernardo Pulci della bucolica virgiliana, otto egloghe del Benivieni, quattro lunghe egloghe del Boninsegni dedicate nel i 468 ad Alfonso e un'ultima egloga dello stesso, dedicata a Lorenzo nel i48I. Si veda F. Battera, L'edizione Miscomini (1482) delle "Bucoliche elegantissimamente composte", in "Studi e problemi di critica testuale», 40, I990, pp. 149-8 ; S. Fornasiero, Presenze (e assenze) della bucolica senese, in La poesia pastorale nel Rinascimento, a cura di S. Carrai, Padova, Antenore, 1998, pp. 58-72.

42. La datazione della prima Arcadia è discussa, ma è probabile che l'opera fosse già finita nel i486. Il ms. più antico è dedicato a Ippolita Sforza, consorte di Alfonso (morta nel I 488). Si veda M. Riccucci, $I l$ neghittoso $e$ il fier connubbio. Storia e filologia nell'Arcadia di Jacopo Sannazaro, Napoli, Liguori, 200 I e la bibliografia citata. 